\title{
COVID-19 en el paciente pediátrico
}

Ma. de los Ángeles del Campo-Martínez ${ }^{1,2 *}$, Berenice Sánchez-Jara¹, Norma C. López-Santiago², Mónica Lozano-Garcidueñas ${ }^{3}$, Janet Soto-Padilla4, A. Mansheca Moreno-González M. Guadalupe Ortiz-Torres MG', Oscar González-Llano ${ }^{6}$, E. Aaron Reyes-Espinoza Lucia M. Muñoz-Juárez-Díaz y Lissette Velázquez-Marmolejo ${ }^{5}$

${ }^{1}$ Unidad Médica de Alta Especialidad Dr. Gaudencio González Garza, Centro Médico Nacional La Raza, Ciudad de México, Méx.; ${ }^{2}$ Instituto Nacional de Pediatría, Ciudad de México, Méx.; ${ }^{3}$ Hospital General de Zona N. ${ }^{\circ}$ 14, Instituto Mexicano del Seguro Social, Guadalajara, Jal.; ${ }^{4}$ Unidad Médica de Alta Especialidad, Centro Médico Nacional de Occidente, Instituto Mexicano del Seguro Social, Guadalajara, Jal.; ${ }^{5}$ Servicio de Hematología, Hospital Infantil de México Federico Gómez, Ciudad de México, Méx.; ${ }^{6}$ Hospital Universitario Dr. José Eleuterio González, Monterrey, N.L.; ${ }^{7}$ Centro Estatal de Cancerología de Durango, Secretaría de Salud, Durango, Durango. México

\section{Resumen}

Este documento fue preparado (en junio de 2020) por y para los profesionales médicos (clínicos y proveedores de salud), y está disponible públicamente para propósitos de recomendaciones relacionada con pacientes pediátricos y enfermedad por coronavirus 2019 (COVID-19). Según datos disponibles hasta la fecha, los niños de todas las edades pueden contraer la COVID-19; sin embargo, tienen mejor pronóstico que los adultos, siendo pocos los casos graves reportados, y los casos leves se recuperan en 1-2 semanas después del inicio de la enfermedad. El objetivo de este documento es aportar peculiaridades que consideramos importantes para el diagnóstico y tratamiento. Debido a los cambios en la actual situación epidemiológica se pueden modificar algunos conceptos y acciones, por lo que la información aquí contenida debe ser adaptada a cada paciente, basada en el juicio clínico, necesidades del paciente y recursos disponibles.

PALABRAS CLAVE: COVID-19. Enfermedad por coronavirus-19. SARS-CoV-2. Síndrome respiratorio agudo severo por coronavirus 2. Paciente pediátrico.

\section{COVID-19 in the pediatric patient}

\section{Abstract}

This document was prepared (June 2020) by and for medical professionals (clinicians and health providers), and it is available as a guideline for pediatric patients with coronavirus disease 2019 (COVID-19). So far, according to available data although children of all ages can acquire COVID-19, they have better outcome than adults, with a little proportion of severe disease, and in mild cases they have a 1 to 2-week recovery after the beginning of the infection. The objective of this document is to provide particularities that we consider important for diagnosis and treatment. Owe to the changing epidemiological situation some concepts and actions can change, so this information must be adapted to each specific case, based on the clinical judgement according to patient requirements and available resources.

KEY WORDS: COVID-19. Coronavirus disease 19. SARS-CoV-2. Severe acute respiratory syndrome coronavirus 2. Pediatric patient.

Correspondencia:

*Ma. de los Ángeles del Campo-Martínez

E-mail: angeleshpinp@yahoo.com.mx

0016-3813/๑ 2021 Academia Nacional de Medicina de México, A.C. Publicado por Permanyer. Este es un artículo open access bajo la licencia CC BY-NC-ND (http://creativecommons.org/licenses/by-nc-nd/4.0/).
Fecha de recepción: 09-09-2020

Fecha de aceptación: 09-02-2021
GacMedMex.2021;157(Supl3):S120-S130

Disponible en PubMed

www.gacetamedicademexico.com 


\section{Introducción}

En diciembre de 2019, las autoridades de salud en Wuhan, China, reportaron casos de neumonía viral severa de origen desconocido que estaban epidemiológicamente vinculados al mercado de mariscos en Wuhan. La secuenciación de muestras del tracto respiratorio reveló un nuevo coronavirus. La enfermedad se denominó posteriormente como enfermedad por coronavirus-19 (COVID-19) y el virus fue nombrado coronavirus 2 del síndrome respiratorio agudo grave (SARS-CoV-2) ${ }^{1}$. El 11 de marzo de 2020, la Organización Mundial de la Salud declaró pandemia y hasta la fecha se han alcanzado casi los seis millones de casos notificados en todo el mundo ${ }^{2}$. En nuestro país se han confirmado, a 24 de junio de 2020, 196,847 casos, de los cuales 5,753 son niños y adolescentes ${ }^{3}$.

Las principales características clínicas en los pacientes adultos son fiebre, tos seca, disnea, mialgia, fatiga, recuento leucocitario normal o leucopenia, y evidencia radiográfica de neumonía ${ }^{4}$. Los pacientes con COVID-19 en estado crítico desarrollan manifestaciones de shock, acidosis metabólica grave, algunos presentan insuficiencia hepática y/o renal además de lesión pulmonar severa. Las citocinas proinflamatorias y quimiocinas, incluido el factor de necrosis tumoral (TNF) alfa, la interleucina $1 \beta$ (IL-1 $1 \beta$ ), la IL-6, el factor estimulante de colonias de granulocitos y monocitos, la proteína 10 inducida por interferón gamma, la proteína-1 monocito quimioatrayente y los macrófagos inflamatorios están significativamente elevados 5 .

En el ámbito pediátrico se ha recogido una proporción de casos en torno al $1 \%$ con respecto a la población general, teniendo la mayoría de nuestros pacientes una sintomatología leve, como fiebre de corta duración ${ }^{6}$. La mayoría de los niños que han requerido atención hospitalaria son los menores de 3 años. En los casos graves presentan disnea con rápida progresión a síndrome de dificultad respiratoria aguda (SDRA), shock séptico, acidosis metabólica, alteraciones en la coagulación y falla orgánica múltiple. En la radiografía de tórax se observan imágenes radiopacas en parches múltiples y cambios intersticiales en la periferia del área pulmonar, consolidaciones pulmonares en casos severos, el derrame pleural se observa raramente. Se considera población pediátrica de alto riesgo a los niños con historia de contacto con casos severos de COVID-19 y los niños con condiciones subyacentes: cardiopatías congénitas, anomalías del tracto respiratorio, desnutrición grave, disminución de los niveles de hemoglobina, inmunodeficiencia y estados de inmunocompromiso, características que presentan con frecuencia los niños con padecimientos hematológicos?

\section{Manifestaciones clínicas}

Desde los reportes iniciales de China se observaron diferencias en las manifestaciones clínicas de COVID-19 en pediatría comparado con el adulto. La primera diferencia fue el número de pacientes infectados. En un estudio de cohorte de 44,672 pacientes solo el $2.1 \%$ fueron menores de 20 años y solo el $1.2 \%$ fueron sintomáticos ${ }^{8}$. Con el paso del tiempo y la diseminación mundial de la enfermedad, se han presentado más casos de pacientes pediátricos, incluyendo neonatos hijos de madres con la infección por SARS-CoV-2, con una evolución a las formas graves más frecuente en menores de 1 año hasta en el $10.6 \%{ }^{9}$.

Es frecuente que en los niños la infección por SARS-CoV-2 no se reconozca debido a que se presenta con manifestaciones leves e inespecíficas tales como hiporreactividad, cefalea, tos, congestión nasal, rinorrea y expectoración; la fiebre puede ser de bajo grado o incluso no estar presente. Diarrea, distensión y dolor abdominal, vómitos y rechazo al alimento son síntomas con los que frecuentemente inician los niños pequeños sin mostrar sintomatología respiratoria ${ }^{10,11}$. De acuerdo con las recomendaciones de la Chinese Medical Association, las manifestaciones clínicas se pueden clasificar según su gravedad ${ }^{12}$.

- Enfermedad leve:

- Asintomáticos o con síntomas respiratorios como congestión nasal y faríngea, rinorrea y fiebre de bajo grado y de corta duración.

- RT-PCR positiva para SARS-CoV-2

- Radiografía de tórax normal.

- Enfermedad moderada:

- Neumonía leve.

- Fiebre, tos, fatiga, cefalea y mialgia.

- Sin otras complicaciones.

- Enfermedad grave. Manifestaciones de enfermedad leve a moderada más alguna manifestación que sugiera progresión de la enfermedad.

- Polipnea (> 70 respiraciones por minuto para $<1$ año, > 50 respiraciones por minuto para > 1 año).

- Hipoxia. 
- Deshidratación, dificultad para la ingesta, disfunción gastrointestinal.

- Daño al miocardio.

- Elevación de las enzimas hepáticas.

- Pérdida de la consciencia, coma, convulsiones.

- Alteraciones de la coagulación, rabdomiólisis o alguna otra manifestación de daño a órganos vitales.

- Enfermedad críticamente grave:

- Progresión rápida.

- Necesidad de ventilación mecánica.

- Choque séptico.

- Falla orgánica múltiple.

- Coagulopatía/trombosis.

- Tormenta de citocinas.

Recientemente, una de las formas más graves es similar a la enfermedad de Kawasaki (EK), con datos de enfermedad sistémica y conocido como síndrome pediátrico de inflamación multisistémica temporalmente asociado a SARS-CoV-2, caracterizado por fiebre persistente, eritema de labios, lengua de "fresa», eritema de mucosa oral y/o faríngea, hiperemia conjuntival, exantema, eritema y edema de manos y pies y descamación periungueal. La presentación puede ser con manifestaciones de choque, o muy similar a EK o bien con fiebre e inflamación persistente sin choque y sin criterios de $\mathrm{EK}^{13}$. No hay evidencia de transmisión vertical de SARS-CoV-2, sin embargo, sí hay reportes de neonatos infectados que se han identificado por alteraciones respiratorias, taquicardia, rechazo al alimento, irritabilidad. En la mayoría de ellos el nacimiento ha sido por parto eutócico, por lo que se ha sugerido que el contagio sucede al contacto con secreciones de la madre enferma ${ }^{14}$.

\section{Alteraciones de laboratorio}

La forma de confirmar la enfermedad es por la identificación de las partículas virales mediante la reacción en cadena de la polimerasa en tiempo real (RT-PCR) en hisopado nasofaríngeo, hoy el estándar de oro, pero es posible recuperarlo de secreción broncoalveolar obtenida por lavado bronquial, esputo, saliva e incluso en heces. El virus tiene un periodo de incubación de 2 a 14 días, aunque la mayoría de los casos se pueden identificar a los 3-7 días ${ }^{10,15}$.

Los exámenes de laboratorio pueden ser totalmente inespecíficos, sin embargo, hay algunas alteraciones que son constantes. Una vez que se ha confirmado el diagnóstico es importante identificar la cuenta de leucocitos y su diferencial; puede haber leucocitosis moderada, sin embargo, es importante identificar la presencia de linfopenia, que se asocia a una reacción inflamatoria pulmonar muy severa que ha inducido apoptosis de los linfocitos, asociado a proteína C reactiva y procalcitonina en valores normales ${ }^{16,17}$. Las plaquetas habitualmente son normales o discretamente disminuidas, pero la asociación a eventos trombóticos arteriales y venosos, ya sea en la microvasculatura o en vasos de mediano y gran calibre, puede llevar a una disminución moderada más alargamiento del tiempo de protrombina, elevación del fibrinógeno y aparición de productos de degradación de la fibrina y dímeros $D(D D)$. Un $D D \geq 6$ veces el valor basal se asocia a un peor pronóstico y si además se añade una elevación de troponina $T$, péptido natriurético y creatina fosfocinasa, coinciden con el peor pronóstico. La elevación de fosfatasa alcalina, deshidrogenasa láctica y alanina aminotransferasa son hallazgos frecuentes asociados a lesión multiorgánica. En los casos graves de COVID-19 la elevación de marcadores inflamatorios como la ferritina resulta en activación de la vasculatura pulmonar y células endoteliales por la IL-1, IL-6, TNF e interferón gamma juegan un rol importante en el proceso tromboinflamatorio pulmonar; todas estas citocinas pueden medirse con fin de establecer medidas terapéuticas específicas de blanco ${ }^{18,19}$.

La radiografía de tórax suele mostrar imágenes en «vidrio despulido» que se refuerzan ya sea mostrando zonas neumónicas localizadas o infiltrados difusos que coinciden con los acúmulos de fibrina intraalveolares. La infiltración inflamatoria subpleural es un hallazgo común en los niños, aun con enfermedad leve.

\section{Hallazgos morfológicos}

En Italia, la Fondazione Policlínico A. Gemelli de Roma ha reportado, tras la observación de frotis de sangre periférica, antes de tratamiento antiviral y/o antiinflamatorio, presencia de neutrofilia en un inicio con cambios morfológicos importantes como granulación tóxica, desviación a la izquierda, formas pseudo-Pelger y células apoptósicas. Estos cambios desaparecen con el inicio de tratamiento (Fig. 1). Tras el inicio del tratamiento, al presentarse una activación linfocitaria importante se evidencian cambios en los linfocitos, con presencia de polimorfismo: linfocitos grandes granulares, atípicos y formas linfoplasmocitoides. En relación con las plaquetas, puede 


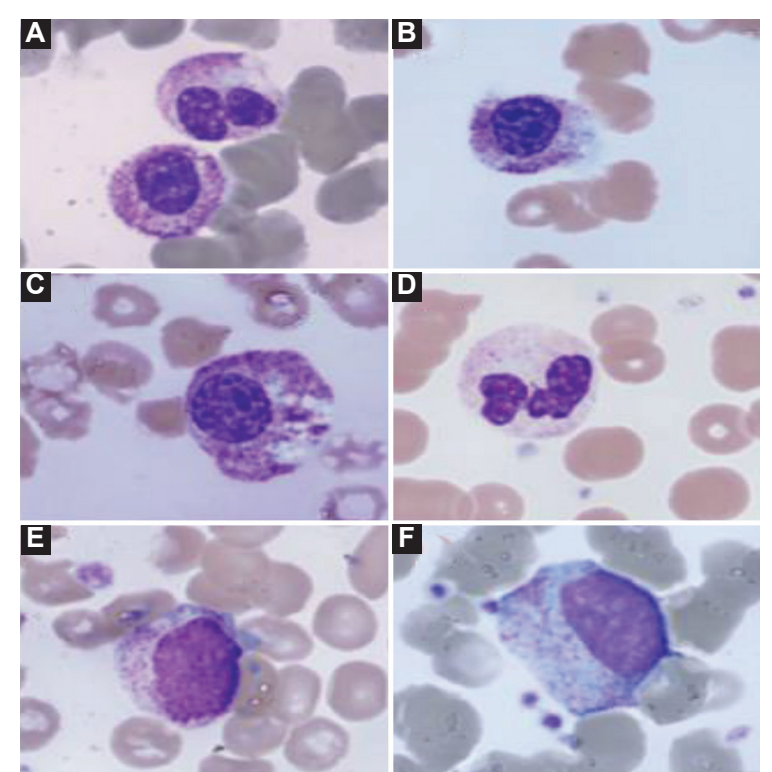

Figura 1. Presencia de anormalidades morfológicas de los neutrófiIos. Los neutrófilos presentan granulaciones tóxicas y vacuolas citoplasmáticas (A, B y C) y, en ocasiones, ausencia de segmentación nuclear con formas de pseudo-Pelger (D), además cayados y desviación a la izquierda con mielocitos y metamielocitos (E y F).

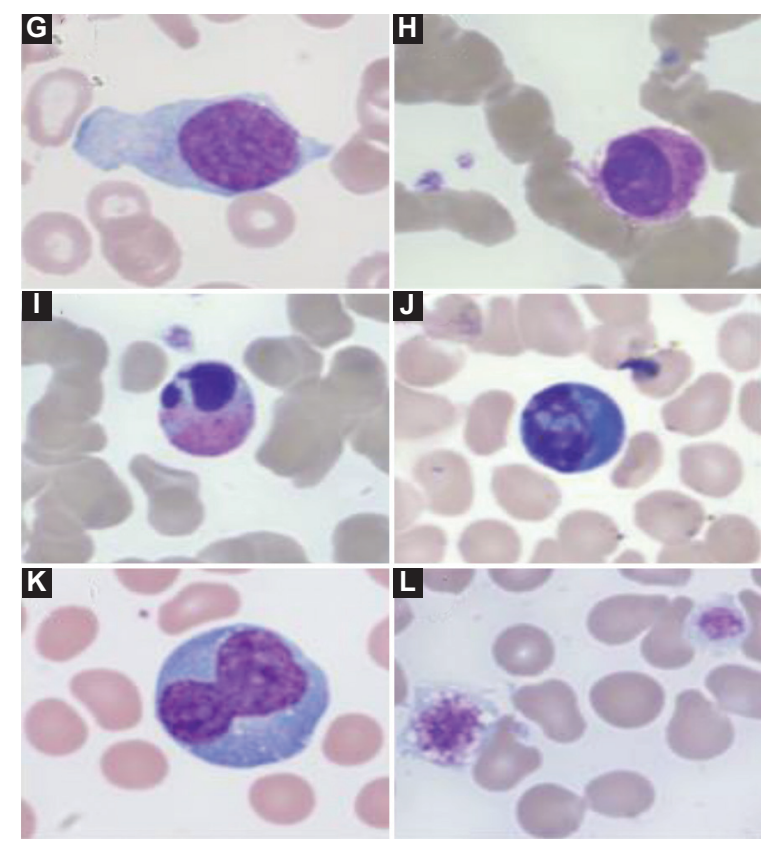

Figura 2. En relación con los linfocitos, se observa polimorfismo importante: células linfocitos grandes granulares, linfocitos atípicos $(\mathrm{G}, \mathrm{H})$, células apoptósicas $(\mathbf{I}, \mathrm{J})$ linfoplasmocitoides $(\mathrm{K})$, megatrombocitos hipercromáticos (L).

presentarse tanto trombocitopenia como trombocitosis, y ambas pueden empeorar el pronóstico (Fig. 2) ${ }^{20}$. La presencia de síndrome de hiperinflamación con

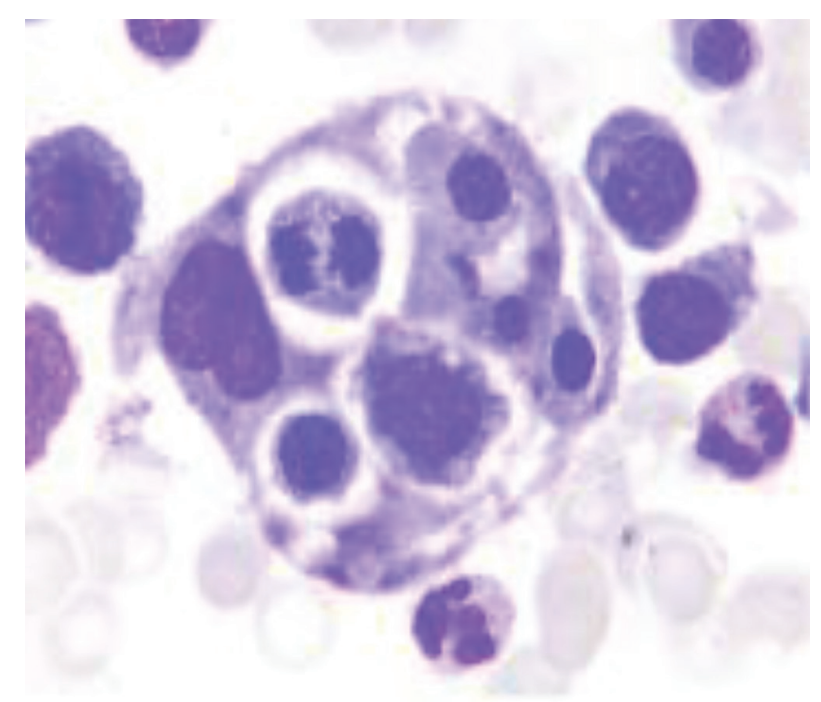

Figura 3. La linfohistiocitosis hemofagocítica es una manifestación menos común del estado hiperinflamatorio.

liberación descontrolada de citocinas inflamatorias puede producir un fenómeno de hemofagocitosis por proliferación de macrófagos (Fig. 3) ${ }^{21}$.

\section{Recomendaciones}

Solicitar biometría hemática diariamente a los pacientes hospitalizados. Revisión de frotis de sangre periférica al ingreso, a los 7 días y al alta del paciente. A todos los pacientes graves deben realizarse pruebas de detección de hiperinflamación.

\section{Sospecha diagnóstica en el paciente pediátrico}

\section{Definición de caso}

La posibilidad de COVID-19 debe considerarse principalmente en niños con fiebre, tos y/o dificultad respiratoria y cualquiera de los criterios de historia epidemiológica (historial de viajes o residencia en comunidades con alto contagio de COVID-19, o de contacto con personas infectadas con SARS-CoV-2 [con PCR positiva] dentro de los 14 días previos al inicio de la enfermedad).

Paciente con enfermedad respiratoria aguda grave que requiere hospitalización en ausencia de un diagnóstico alternativo que explique la presentación clínica y/o paciente con una condición médica crónica subyacente (cardiopatía congénita, enfermedad pulmonar, renal o hepática crónica, en tratamiento 


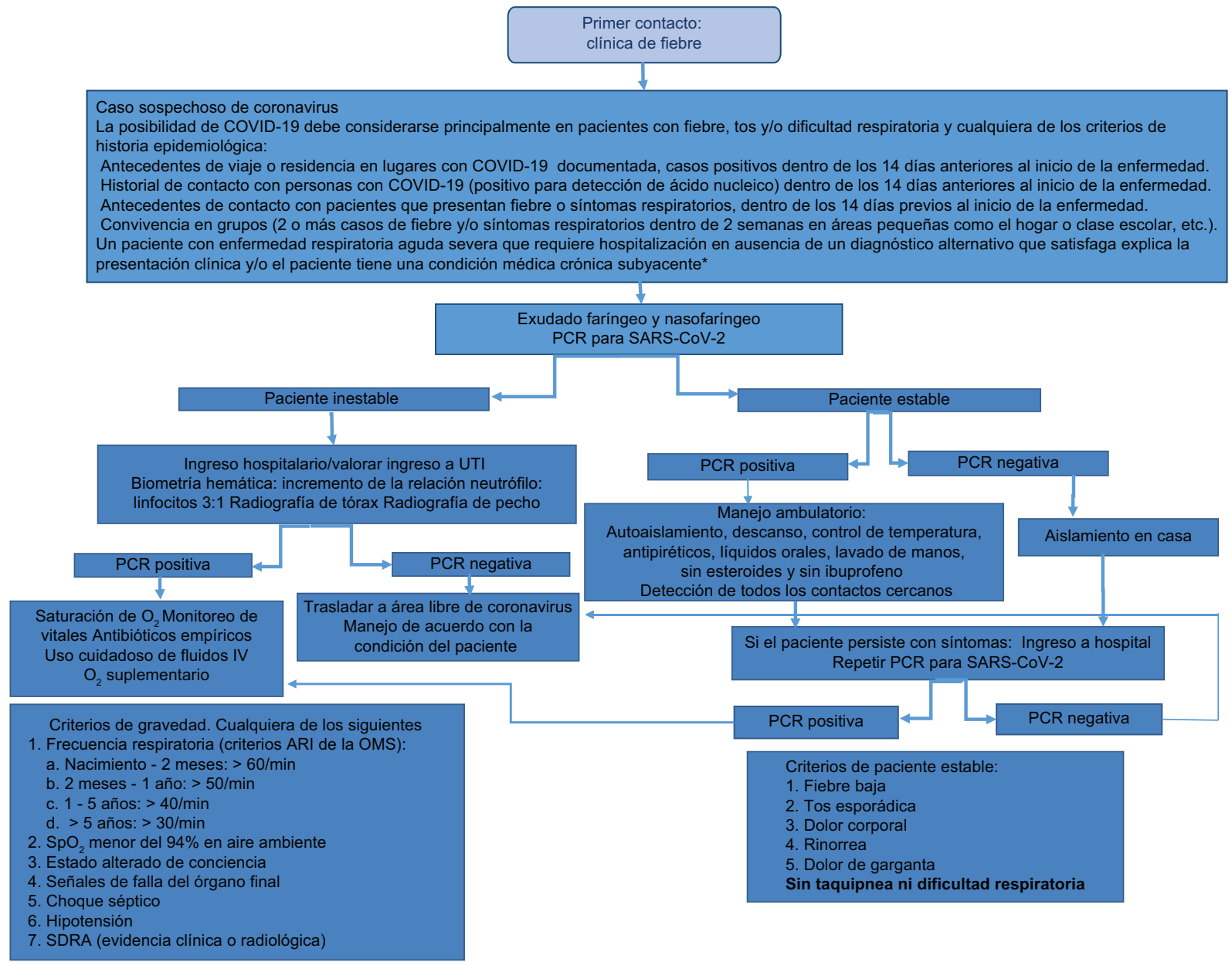

Figura 4. Algoritmo para sospecha diagnóstica en el paciente pediátrico.

*Cáncer, inmunosupresión, enfermedades crónicas

COVID-19: enfermedad por coronavirus 2019; SARS-CoV-2: coronavirus 2 del síndrome respiratorio agudo grave; UTI: unidad de terapia intensiva; PCR: reacción en cadena de la polimerasa; ARI: infección respiratoria aguda; OMS: organización Mundial de la Salud; SDRA: síndrome de dificultad respiratoria aguda; $\mathrm{SpO}_{2}$ : saturación de oxígeno.

inmunosupresor o inmunocomprometido, por ejemplo, estado postrasplante) (Fig. 4) $)^{22-24}$.

\section{Criterios de sospecha}

- Un niño que presenta fiebre persistente, inflamación (neutrofilia, PCR elevada y linfopenia) y evidencia de disfunción de uno o varios órganos (shock, cardiaco, trastorno respiratorio, renal, gastrointestinal o neurológico) con características adicionales. Esto puede incluir a niños que cumplan criterios completos o parciales para EK.

- Exclusión de cualquier otra causa microbiana, incluyendo sepsis bacteriana, estafilococos o síndrome de choque estreptocócico, e infecciones asociadas con miocarditis, como el enterovirus.

- Las pruebas de PCR de SARS-CoV-2 pueden ser positivas o negativas ${ }^{23-25}$.

Es importante para el diagnóstico de un caso sospechoso de COVID-19 la realización de una historia clínica detallada y usar juicio clínico adecuado. Las coinfecciones pueden ser más comunes en los niños. Los niños inmunocomprometidos pueden estar predispuestos a infecciones graves por coronavirus. La presencia de un copatógeno y factores del huésped, incluido un estado inmunocomprometido, se asociaron con un mayor riesgo de gravedad. Reconocer los factores de riesgo de enfermedad respiratoria grave podría ayudar en la estratificación del riesgo ${ }^{26}$. 


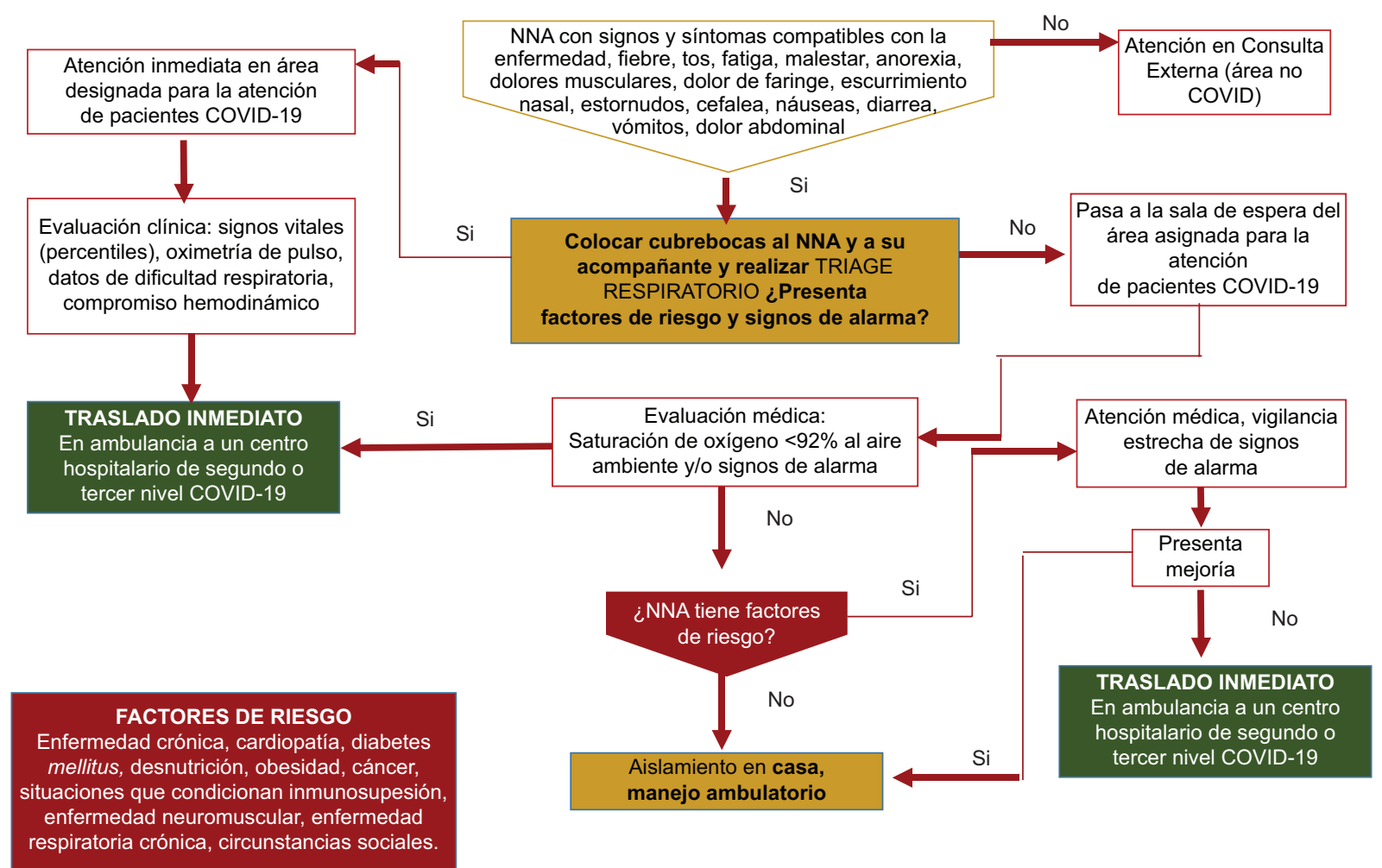

Figura 5. Algoritmo para tratamiento de pacientes ambulatorios.

COVID-19: enfermedad por coronavirus 2019; NNA: niños, niñas y adolescentes.

\section{Tratamiento de pacientes ambulatorios (Fig. 5)}

\section{Educación de los responsables sobre los datos de alarma}

- Asegurar hidratación adecuada.

- En caso de fiebre: utilizar medios físicos y/o paracetamol 10-15 mg/kg/dosis cada 6 u 8 horas.

- Los lactantes deben continuar con lactancia materna y alimentación propia para la edad. Si presentan datos de alarma acudir inmediatamente a la unidad de salud. Consulta de seguimiento a las 24 horas (niños, niñas, adolescentes con factores de riesgo).

\section{Signos de alarma}

- Datos de dificultad respiratoria:

- Oximetría de pulso menor a 92\% respirando al aire ambiente.

- Taquipnea $0<2$ meses $\geq 60$ rpm; 2-11 meses, $\geq 50 \mathrm{rpm} ; 1-4$ años, $\geq 40 \mathrm{rpm}$; $1-4$ años, $\geq 40$ rpm; mayor a 5 años $>30$ rpm.
- Datos de hipoperfusión tisular: alteración en el estado de alerta (irritabilidad), Ilenado capilar prolongado, palidez, coloración marmórea, extremidades frías.

- Hipotensión arterial (presión arterial sistólica $<$ percentil 50 > a 2 desviaciones estándar por debajo de lo normal para la edad).

- Vómitos o diarrea persistente, confusión, letargo, descontrol glucémico ${ }^{27-29}$.

\section{Tratamiento de pacientes hospitalizados} (Fig. 6)

La infección por SARS-CoV-2 ha contribuido a una mortalidad significativa de forma mundial, afectando hasta en un $1.7 \%$ a pacientes pediátricos $(<18 \text { años })^{30}$. Según reportes en China, en niños la COVID-19 se presenta de forma menos grave ${ }^{31}$, sin embargo, las alteraciones en la coagulación se han descrito como una complicación asociada frecuente y de gran importancia $^{32}$. En estudios recientes, entre el 20 y el $55 \%$ de los pacientes hospitalizados secundario a infección por SARS-CoV-2 presentaron evidencia de alteraciones hematológicas, siendo la más importante la 

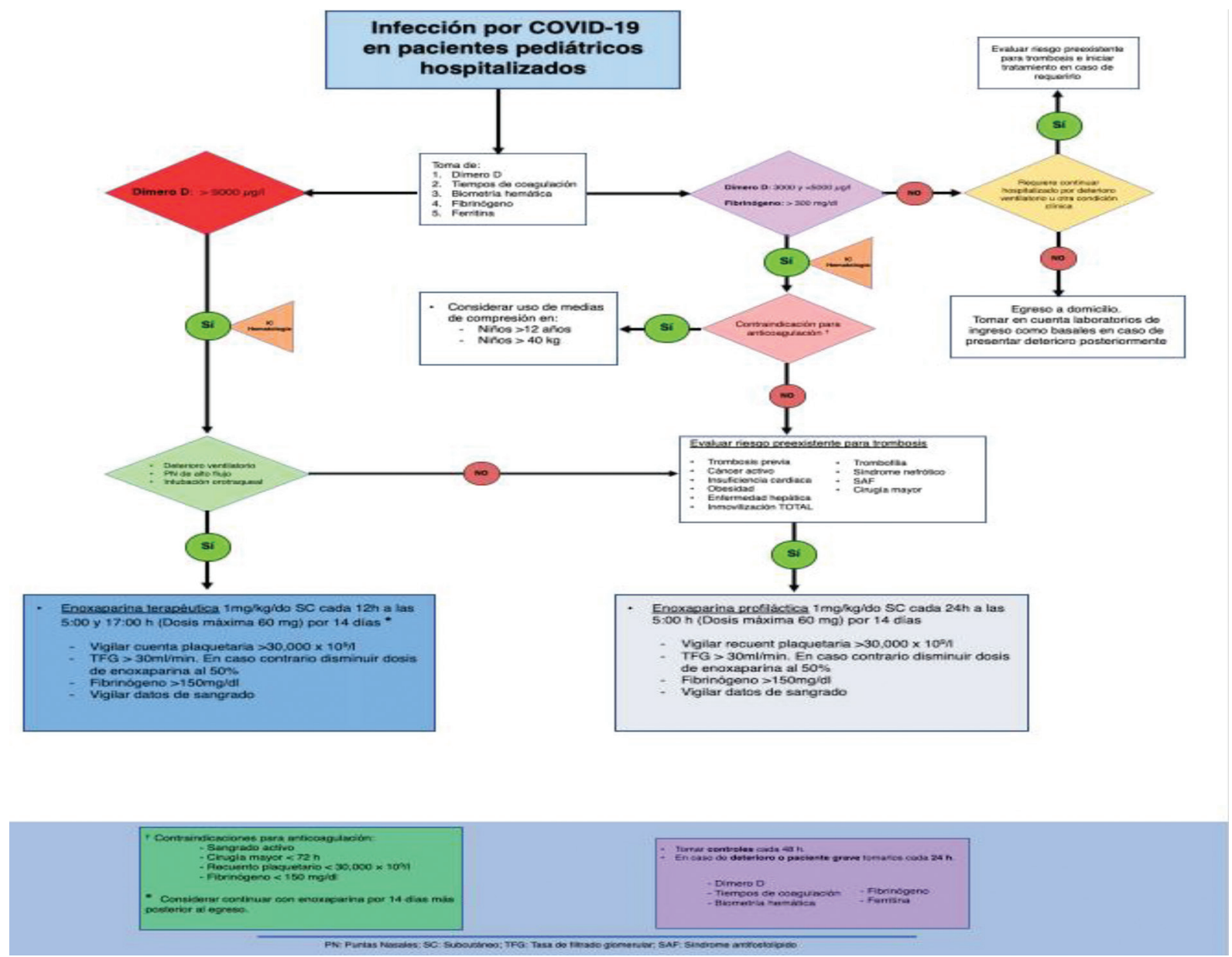

Figura 6. Algoritmo para tratamiento de pacientes hospitalizados.

${ }^{*}$ Considerar continuar con enoxaparina por 14 días más posterior al egreso.

${ }^{+}$Contraindicaciones para anticoagulación: sangrado activo, cirugía mayor $<72 \mathrm{~h}$, plaquetas $<30,000$ x 10\%/, fibrinógeno $<150 \mathrm{mg} / \mathrm{dl}$.

PN: puntas nasales; SC: subcutáneo; TFG: tasa de filtrado glomerular; IC: insuficiencia cardiaca; SAF: síndrome antifosfolípido.

coagulación intravascular diseminada de predominio protrombótica y encontrando una incidencia del $25 \%$ de trombosis venosa profunda en estos pacientes ${ }^{33}$. Actualmente están surgiendo guías internacionales sobre el papel potencial de la anticoagulación de forma profiláctica para prevenir esta complicación ${ }^{34}$. Las guías provisionales para el reconocimiento y tratamiento de coagulopatía por COVID-19 de la International Society on Thrombosis and Haemostasis (ISTH) recomiendan que en pacientes con valores de DD elevados de tres a cuatro veces el valor normal y en pacientes pediátricos (menores a 21 años) con valores de DD mayores a 5,000 $\mu \mathrm{g} / \mathrm{l}$ se deberá iniciar terapia anticoagulante de forma profiláctica o terapéutica según las condiciones clínicas del paciente, ya que el aumento de DD representa aumento de la generación de trombina y es predictor del desarrollo del SDRA.
En cuanto al tratamiento, la heparina de bajo peso molecular (HBPM) es la de elección, ya que se encuentra ampliamente disponible y se ha demostrado que además de funcionar como profilaxis y tratamiento para trombosis, tiene propiedades antiinflamatorias que pueden ser un beneficio adicional en la COVID-19 ante un incremento importante de las citocinas proinflamatorias ${ }^{35}$.

\section{Anticoagulación en niños con infección por SARS-CoV-2}

Los eventos tromboembólicos en niños tienen una baja incidencia. Estudios realizados por Raffini, et al. han reportado una incidencia anual de 34 a 58 por cada 10,000 admisiones hospitalarias y de 5.1 por cada 100,000 en neonatos. Cuando ocurren 
generalmente se presentan en pacientes hospitalizados con factores de riesgo como las infecciones graves, estados proinflamatorios, cardiopatía, cáncer, deshidratación, eventos quirúrgicos, politraumatismo, inmovilidad, uso de accesos vasculares y trombofilia hereditaria 0 adquirida ${ }^{36}$.

Debido a que la infección por SARS-CoV-2 se trata de un nuevo padecimiento, la información reportada está sujeta a constante actualización a medida que avanzan los reportes de casos. Las recomendaciones de la terapia antitrombótica han sido extrapoladas de las de adultos. Sin embargo, el tratamiento anticoagulante en neonatos y niños difiere en diversos aspectos, debido a variaciones ontogénicas de la hemostasia que afectan tanto a la fisiopatología de los procesos trombóticos como a la respuesta a los fármacos antitrombóticas por diferencias farmacocinéticas y farmacodinámicas.

Las HBPM se han convertido en el anticoagulante de elección en pediatría. Tanto para profilaxis como para tratamiento, la dosis se ajusta de acuerdo con la indicación clínica y la edad (Tabla 1).

Las indicaciones de anticoagulación sugeridas en pacientes con COVID-19 se muestran en la tabla 2.

Cuando se utiliza anticoagulación a dosis profilácticas, se sugiere emplearla hasta que el paciente sea egresado de la unidad de terapia intensiva, es decir, cuando se encuentre estable hemodinámicamente y sin afectación de los parámetros hemostásicos. Si la anticoagulación fue terapéutica, el tiempo de anticoagulación va de 3 a 6 meses según el evento subyacente que provocó la trombosis o la resolución del estado de hipercoagulabilidad ${ }^{37-40}$.

\section{Consideraciones especiales para pacientes pediátricos con enfermedades hematológicas}

La mayor parte de las recomendaciones para niños con enfermedades hematológicas han sido presentadas por hospitales y organizaciones del primer mundo (International Society of Paediatric Oncology, Grupo de Oncología Infantil, International Pediatric Surgical Oncology Association, St. Jude Global), por lo que es posible que no se puedan llevar a cabo plenamente por las circunstancias que vivimos en nuestro país, además, estas recomendaciones no cuentan por razones obvias con el mejor nivel de evidencia ${ }^{41}$.
Tabla 1. En el paciente pediátrico la dosis de heparina de bajo peso molecular (el anticoagulante de elección) se ajusta de acuerdo con la indicación clínica y la edad

\begin{tabular}{c|c|c}
$\begin{array}{l}\text { Dosis de } \\
\text { enoxaparina } \\
\text { según la edad }\end{array}$ & Tratamiento & Profilaxis \\
\hline $\begin{array}{c}\text { Paciente } \\
<2 \text { meses }\end{array}$ & $1.5 \mathrm{Ul} / \mathrm{kg} / \mathrm{do}$ cada $12 \mathrm{~h}$ & $0.75 \mathrm{Ul} / \mathrm{kg} / \mathrm{do}$ cada $12 \mathrm{~h}$ \\
$>2$ meses & $1 . \mathrm{Ul} / \mathrm{kg} / \mathrm{do}$ cada $12 \mathrm{~h}$ & $0.5 \mathrm{Ul} / \mathrm{kg} / \mathrm{do}$ cada $12 \mathrm{~h}$
\end{tabular}

Las guías hacen hincapié en cuidar tanto la salud de los niños y sus familias como la del personal de salud involucrado en su atención. La coincidencia más importante de la mayor parte de lo publicado es que hasta donde sea posible y de acuerdo con las condiciones de cada país y/o centro de atención, los procesos de diagnóstico, tratamiento y medidas de soporte de los niños con cáncer no se vean afectadas. En nuestro país, como todos ustedes saben, habrá que agregar el muy complicado acceso a los diferentes fármacos quimioterapéuticos.

No obstante las recomendaciones internacionales, los datos obtenidos mediante encuestas realizadas en Latinoamérica muestran un retraso en consultas y principalmente en cirugías, tratamientos de radioterapia y procedimientos ambulatorios ${ }^{42}$.

Parece razonable sugerir que las modificaciones que pudieran establecerse se lleven a cabo de manera colegiada entre los miembros del equipo. Los cambios y diferimientos serán más frecuentemente realizados en los procedimientos, consultas y tratamiento de pacientes con padecimientos no urgentes.

Imposible detallar en un espacio tan breve recomendaciones precisas a propósito de la emergencia de la pandemia en nuestros pacientes, la información publicada por médicos italianos en la región más afectada de ese país muestra datos de mucha importancia que nos pueden ser de utilidad ${ }^{43}$.

Finalmente, en niños con leucemias y linfomas se recomienda, como se mencionó arriba, llevar a cabo los procedimientos diagnósticos y de tratamiento de la manera más oportuna posible y realizando el menor número de modificaciones de acuerdo con las necesidades y carencias de cada centro. En las circunstancias actuales de la pandemia en nuestro país, con la problemática de abasto y la muy probable sobreocupación de camas hospitalarias, estaremos enfrentando seguramente todos nosotros un reto mayúsculo en los siguientes meses. 
Tabla 2. Indicaciones de anticoagulación sugeridas en pacientes pediátricos con enfermedad por coronavirus 2019 (COVID-19)

\begin{tabular}{|c|c|}
\hline \multicolumn{2}{|l|}{ Recomendaciones para anticoagulación en niños y COVID-19 } \\
\hline aciente con COVID-19 y clasificación clínica de infección grav & y/o algunas de las siguientes condiciones \\
\hline $\begin{array}{l}\text { Indicaciones para prevención tromboembolia (dosis } \\
\text { profilácticas) } \\
\text { - Todo paciente con COVID-19 grave, ingresado a unidad de } \\
\text { cuidados intensivos } \\
\text { - Cuadro clínico similar a enfermedad de Kawasaki en el que se } \\
\text { documente afección cardiaca } \\
\text { - Trombofilia hereditaria y/o adquirida }\end{array}$ & $\begin{array}{l}\text { Indicaciones para dosis terapéutica de anticoagulantes } \\
\text { - Cuadro sugestivo de síndrome de shock tóxico o síndrome } \\
\text { inflamatorio multisistémico asociado a COVID-19 } \\
\text { - Evidencia por estudio de imagen de tromboembolia venosa } \\
\text { o arterial en extremidades, microangiopatía, EVC o trombosis } \\
\text { asociada a catéter central o tromboembolia pulmonar } \\
\text { - Miocardiopatía, valvulopatía, prótesis valvulares } \\
\text { - Pacientes que ya recibían anticoagulación oral previo a la } \\
\text { infección se sugiere continuar de manera parenteral } \\
\text { - Falla multiorgánica } \\
\text { - Coagulación intravascular diseminada } \\
\text { - Otros: PCR > 15, dímero-D > 1,500 ng/ml o elevación de } 4 \text { veces } \\
\text { su nivel basal en ausencia de otro hallazgo, IL-6 }>40 \text {, ferritina > } \\
\text { 1,000, linfopenia }<800\end{array}$ \\
\hline
\end{tabular}

EVC: evento vascular cerebral; PCR: proteína C reactiva.

\section{Conclusiones}

La infección por SARS-CoV-2 ha causado miles de muertes en todo el mundo. Los niños en todas las edades son susceptibles de contraer la infección y aquellos con enfermedades subyacentes son quienes más comúnmente presentan manifestaciones gra$v \mathrm{ve}^{44}$. Los síntomas son muy variables en este grupo, presentándose portadores asintomáticos, síntomas respiratorios leves a moderados, con tos seca asociada o no a fiebre, fatiga, congestión nasal, dolor abdominal, vómitos, etc. En más del $50 \%$ la fiebre con tos y respiración entrecortada se observan al inicio ${ }^{45}$. El número de pacientes graves es significativamente menor al observado en adultos, teniéndose reportes de que solo el $5 \%$ de los infectados presentan hipoxemia o disnea, con progresión a síndrome de insuficiencia respiratoria o falla multiorgánica en solo el $0.6 \%^{9,46}$. Otros autores han reportado frecuencias más altas de complicaciones, con desarrollo de síndrome inflamatorio multisistémico ${ }^{22}$, síndrome hemofagocítico secundario, etc. Existen diferentes hipótesis para explicar la diferencia en la presentación y gravedad en niños, como son las diferencias del sistema inmunitario (madurez), competición e interacciones virales en la mucosa de la vía aérea que pudiera contener el crecimiento del SARS-CoV-2, diferencias en la expresión del receptor de la enzima convertidora de angiotensina (necesaria para la fijación del virus), y las diferencias en las características fenotípicas y funcionales del sistema inmunitario, pues con la edad existe disminución progresiva de la capacidad inmunitaria que conlleva respuestas inmunológicas más débiles y altera la capacidad de responder a nuevos estímulos $^{47,48}$. El diagnóstico se establece mediante las características epidemiológicas y clínicas, empleo de la prueba de RT-PCR para SARS-CoV-2 y empleo de pruebas serológicas (inmunoglobulina [lg] $\mathrm{G}$ e $\operatorname{lgM})^{44}$, encontrándose que a diferencia de los adultos, la tomografía muestra patrones atípicos, con una extensión de la imagen de vidrio despulido más delimitada y rara vez engrosamiento septal interlobar ${ }^{49}$. Debido a las múltiples complicaciones en los grupos de pacientes que desarrollan la enfermedad, se han realizado recomendaciones de acuerdo con situaciones específicas, como en los recién nacidos de madres con infección ${ }^{50,51}$, en pacientes con neoplasias hematológicas ${ }^{41}$, en niños y adolescentes con leucemia ${ }^{52}$, en pacientes pediátricos con diferentes tipos de neoplasias ${ }^{53}$, pacientes con lesión renal aguda secundaria ${ }^{54}$, pacientes con diabetes ${ }^{55}$, pacientes con enfermedades reumatológicas y terapias biológicas ${ }^{56}$, pacientes con hemofilia ${ }^{57}$, etc. Para los pacientes con manifestaciones graves se han empleado diferentes estrategias terapéuticas, como se ha mencionado en el presente artículo, teniéndose mayor experiencia en pacientes adultos por las cuestiones epidemiológicas ya comentadas. El uso de remdesivir ha arrojado resultados iniciales satisfactorios $^{58}$, para el control de la tormenta de citocinas y el síndrome hemofagocítico secundario se ha empleado ruxolitinib, mostrando utilidad al mejorar variables hematológicas y radiológicas ${ }^{59}$, y estudios clínicos con empleo de anakinra, tozilizumab, siltuximab y sarilumab están en desarrollo ${ }^{60}$ : Las recomendaciones para anticoagulación se pueden extrapolar a la 
edad pediátrica, considerándose útil solo en aquellos pacientes hospitalizados, con enfermedad moderada a grave, con factores de riesgo protrombóticos y con un incremento significativo del DD ${ }^{44,61}$. Existen aún muchas incógnitas en el tratamiento adecuado de la infección por SARS-CoV-2, siendo indispensable el manejo multidisciplinario, considerando los múltiples órganos y sistemas que pueden afectarse en la presentación moderada y grave de la enfermedad. La intervención oportuna y apropiada del médico pediatra y de los especialistas en diversas ramas de la pediatría (terapia intensiva, neumología, infectología, hematología, inmunología, etc.) son esenciales para lograr un mejor pronóstico.

\section{Conflicto de intereses}

Los autores declaran no tener ningún conflicto de intereses.

\section{Financiamiento}

La presente investigación no ha recibido ninguna beca específica de agencias de los sectores público, comercial, o sin ánimo de lucro.

\section{Responsabilidades éticas}

El presente artículo representa una revisión de la literatura actualmente disponible sobre el tema; los autores declaran que no se ha realizado ningún estudio clínico o de experimentación en seres humanos o animales. Se incluyen todas las fuentes de información.

\section{Bibliografía}

1. Kam KQ, Yung CF, Cui L, Tzer Pin Lin R, Mak TM, Maiwald M, et al. A well infant with coronavirus disease 2019 with high viral load. Clin Infect Dis. 2020;71(15):847-9.

2. Zheng $\mathrm{F}$, Liao $\mathrm{C}$, Fan $\mathrm{QH}$, et al. Clinical characteristics of children with coronavirus disease 2019 in Hubei, China. Curr Med Sci. 2020;40(2):275280. doi:10.1007/s11596-020-2172-

3. Secretaría de Salud. Gobierno de México.

4. Tang N, Li D, Wang X, Sun Z. Abnormal coagulation parameters are associated with poor prognosis in patients with novel coronavirus pneumonia. J Thromb Haemost. 2020;18(4):844-847. doi:10.1111/jth.14768

5. Li H, Liu L, Zhang D, Chen HB, Zhao XG, Xie ZG, et al. SARS-CoV-2 and viral sepsis: observations and hypotheses. Lancet. 2020;395(10235):1517-20.

6. Sanders JM, Monogue ML, Jodlowski TZ, Cutrell JB. Pharmacologic treatments for coronavirus disease 2019 (COVID-19): A review. JAMA. 2020;323(18):1824-36.

7. Ludvigsson JF. Systematic review of COVID-19 in children shows milder cases and a better prognosis than adults. Acta Paediatr. 2020;109(6):1088-95.

8. Novel Coronavirus Pneumonia Emergency Response Epidemiology Team. The epidemiological characteristics of an outbreak of 2019 nove coronavirus diseases (COVID-19) in China. Zhonghua Liu Xing Bing Xue Za Zhi. 2020;41:145-51
9. Dong $Y$, Mo X, Hu Y, Qi X, Jiang F, Jiang Z, et al. Epidemiological characteristics of 2143 pediatric patients with 2019 coronavirus disease in China. Pediatrics. 2020;145(6): e20200702.

10. De Rose DU, Piersigilli F, Ronchhetti MP, Santisi A, Bersani I, Dotta A, et al. Novel coronavirus disease (COVID-19) in newborns and infants: what we know so far. Ital J Pediatr. 2020;46(1):56-63.

11. Cao $Q$, Chen YC, Chen LC, Chiu CH. SARS-CoV-2 infection in children: transmission dynamics and clinical characteristics. J Formosan Med Association. 2020;119:670-3.

12. Qiu H, Wu J, Hong L, Luo Y, Song Q, Chen D. Clinical and epidemiological features of 36 children with coronavirus disease 19 (COVID 19) in Zhejiang. China: an observational cohort study. Lancet Infect Dis. 2020;20:689-96

13. Whittaker E, Bamford A, Kenny J, Kaforou M, Jones CE, Shah P, et al. Clinical characteristics of 58 children with pediatric inflammatory multisystem syndrome temporally associated with SARS-CoV-2. JAMA 2020;324(3):259-69.

14. Hong $\mathrm{H}$, Wang $\mathrm{Y}$, Chung HT, Chen CJ. Clinical characteristics of nove coronavirus disease 2019 (COVID-19) in newborns, infants and children. Pediatr Neonatol. 2020;61:131-2.

15. Zhu N, Zhang D, Wang W, Li X, Yang B, Song J, et al. A novel coronavirus from patients with pneumonia in China, 2019. N Engl J Med. 2020;382(8):727-33

16. Iba T, Levy JH, Levi M, Connors JM, Thachil J.Coagulopathy of coronavirus disease 2019. Crit Care Med. 2020;48(9):1358-64.

17. Wang L, Shi $Y$, Xiao T, Fu J, Feng $X, M u ~ D$, et al. Chinese expert consensus on the perinatal and neonatal management for the prevention and control of the 2019 novel coronavirus infection (first edition). Ann Transl Med. 2020:8:47.

18. McGanagle D, O'Donnell JS, Sharif K, Emery P, Bridgewood C. Immune mechanisms of pulmonary intravascular coagulopathy in COVID-19 pneumonia. Lancet Rheumatol. 2020;2(7):e437-e445.

19. Levi M, van der Poll T. Coagulation and sepsis. Thromb Res. 2017;149: 38-44.

20. Zini G, Bellesi S, Ramundo F, d'Onofrio G. Morphological anomalies of circulating blood cells in COVID-19. Am J Hematol. 2020;95(7):870-2.

21. Dewaele K, Claeys R. Hemophagocytic lymphohistiocytosis in SARSCoV-2 infection. Blood. 2020;135(25):2323.

22. Cheung EW, Zachariah P, Gorelik M, Boneparth A, Kernie SG, Orange JS, et al. Multisystem inflammatory syndrome related to COVID-19 in previously healthy children and adolescents in New York City. JAMA. 2020;324(3):294-6.

23. Tagarro A, Epalza C, Santos M, Sanz-Santaeufemia FJ, Otheo E, Moraleda C, et al. Screening and severity of coronavirus disease 2019 (COVID-19) in children in Madrid, Spain. JAMA Pediatr. 2020 Apr 8;e201346. doi: 10.1001/jamapediatrics.2020.1346. Online ahead of print.

24. Sadiq M, Saleem M, Yasin M, Junaid A, Aa Bari A. Handbook of pediatric COVID - 19 \& standard operating procedures. Hand B Pediatr COVID-19. 2020:2:1-64

25. Multisystem inflammatory syndrome in children and adolescents with COVID-19: scientific brief, 15 May 2020 [Internet]. World Health Organization; 15/05/2020. Disponible en: WHO/2019-nCoV/Sci_Brief/Multisystem Syndrome_Children/2020

26. Ogimi C, Englund JA, BradfordC, Qin X, Boeckh M, Waghmare A. Characteristics and outcomes of coronavirus infection in children: The role of viral factors and an immunocompromised state hcov infection in immunocompromised children. J Pediatric Infect Dis Soc. 2019;8(1):21-8.

27. Secretaría de Salud. Dirección General de Epidemiología. Lineamiento Estandarizado para la Vigilancia Epidemiológica y por Laboratorio de la Enfermedad Respiratoria Viral. México: Secretaría de Salud, Dirección General de Epidemiología; abril de 2020.

28. Manejo clínico del COVID-19: atención hospitalaria. Documento TécnicoCoordinación: Centro de C oordinación de Alertas Sanitarias. Dirección General de Salud Pública, Calidad e Innovación. Gobierno de España. Ministerio de Salud. Se puede consultar en: https://www.mscbs. gob.es/profesionales/saludPublica/ccayes/alertasActual/nCov/documentos/Protocolo manejo clínico ah Covid-19.pdf

29. Secretaría de Salud. Manual de Enfermedades Respiratorias 2012. Prevención, diagnóstico y tratamiento. México: Secretaría de Salud; 2012.

30. CDC COVID-19 Response Team. Coronavirus Disease 2019 in Children, United States, February 12-April 2, 2020. MMWR Morb Mortal Wkly Rep. 2020;69(14):422-6.

31. Zimmermann P, Curtis N. COVID-19 in children, pregnancy and neonates: A review of epidemiologic and clinical features. Pediatr Infect Dis J. 2020;39:469-77

32. Thachil J. Interim guidance on recognition and management of coagulopathy in COVID-19. J Thromb Haemost. 2020;18:1023-6.

33. Cui S, Chen S, Li X, Liu S, Wang F. Prevalence of venous thromboembolism in patients with severe novel coronavirus pneumonia. J Thromb Haemost. 2020;18(6):1421-4.

34. Lee S, Fralick M, Sholzberg M. Coagulopathy associated with COVID-19. CMAJ. 2020;192:E583 
35. Poterucha TJ, Libby P, Goldhaber SZ. More than an anticoagulant: do heparins have direct anti-inflammatory effects? Thromb Haemost. 2017; 117(3):437-44

36. Hennon R, Penque M, Abdul-Aziz R, Alibrahim OS, McGreevy MB, Prout AJ, et al. COVID-19 associated Multisystem Inflammatory Syndrome in Children (MIS-C) guidelines; a Western New York approach. Prog Pediatr Cardiol. 2020 May 23:101232. doi: 10.1016/j.ppedcard.2020.101232. Online ahead of print.

37. Howard SC. Pediatric thromboembolism [Internet]. Medscape; 23/05/2019. Disponible en: https://emedicine.medscape.com/article/959501-overview

38. Liguoro I, Pilotto Ch, Bonannil M, Ferrari ME, Pusiol A, Nocerino A et al. SARS-COV-2 infection in children and newborns: a systematic review, May 2020. Eur J Pediatr. 2020;179(7):1029-46.

39. COVID-19 Treatment Guidelines Panel. Coronavirus Disease 2019 (CO VID-19) Treatment Guidelines [Internet]. National Institutes of Health. Disponible en: https://www.covid19treatmentguidelines.nih.gov

40. He Y, Lin Z, Tang D, Yang Y, Wang T, Yang M. Strategic plan for management of COVID-19 in pediatric hematology and oncology departments. Lancet Haematol. 2020;7(5):e359-e362.

41. Sullivan M, Bouffet E, Rodriguez-Galindo C, Luna-Fineman S, Khan MS Kearns $P$, et al. The COVID-19 pandemic: A rapid global response for children with cancer from SIOP, COG, SIOP-E, SIOP-PODC, IPSO, PROS, CCI, and St Jude Global. Pediatr Blood Cancer. 2020;67(7):e28409.

42. Vasquez L, Sampor C, Villanueva G, Maradiegue E, Garcia-Lombardi M, Gomez-García W, et al. Early impact of the COVID-19 pandemic on paediatric cancer care in Latin America. Lancet Oncol. 2020;21(6):753-5.

43. Balduzzi A, Brivio E, Rovelli A, Rizzari C, Gasperini S, Melzi ML, et al. Lessons after the early management of the COVID-19 outbreak in a pediatric transplant and hemato-oncology center embedded within a COVID-19 dedicated hospital in Lombardia. Bone Marrow Transplant. 2020;55(10):1900-5.

44. Shen $\mathrm{KL}$, Yang $\mathrm{YH}$, Jiang RM, Wang TY, Zhao DC, Jiang Y, et al. Updated diagnosis, treatment and prevention of COVID-19 in children: experts' consensus statement (condensed versión of the second edition). World J Pediatr. 2020;16(3):232-9.

45. Kammoun R, Masmoudi K. Paediatric aspects of COVID-19: An update. Respir Med Res. 2020;78:100765

46. Cruz AT, Zeichner SL. COVID-19 in children: Initial characterization of the pediatric disease. ediatrics. 2020;145(6):e20200834.

47. Brodin P. Why is COVID so mild in children. Acta Paediatr. 2020;109(6):1082-3.

48. Ruggiero A, Attina G, Chiaretti A. Additional hypotheses about why COVID-19 is milder in children tan adults. Acta Paediatr. 2020;109(8):1690

49. Duan Yn, Zhu Yq. Tang LI, Qin J. CT features of novel coronavirus pneumonia (COVID-19) in children. Eur Radiol. 2020;30(8):4427-33.
50. Amatya S, Corr T, Gandhi C, Glas K, Kresch M, Mujsce D, et al. Management of newborns exposed to mothers with confirmed or suspected COVID-19. J Perinatol. 2020;40:987-96.

51. Lavizzari A, Klingenberg C, Profit J, Zupancic J, Davis A, Mosca F, et al. International comparison of guidelines for managing neonates at the early phase of the SARS-CoV-2 pandemic. Pediatr Res. 2020 Jun 15. doi: 10.1038/s41390-020-0976-5. Online ahead of print.

52. He W, Chen L, Chen L, Yuan G, Fang Y, Chen W, et al. COVID-19 in persons with haematological cancers. Leukemia. Leukemia. 2020;34(6):1637-45

53. Baruchel A, Bertrand Y, Boissel N, Brethon B, Ducassou S, Gandemer V, et al. COVID-19 and acute lymphoblastic leukemias of children and adolescents: First recommendations of the Leukemia committee of the French Society for the fight against Cancers and Leukemias in children and adolescents (SFCE). Bull Cancer. Bull Cancer. 2020;107(6):629-32.

54. Ronco C, Reis T, Husain-Syed F. Management of acute kiney injury in patients with COVID-19. Lancet Respir Med. Lancet Respir Med. 2020;8(7):738-42

55. Bornstein S, Rubino F, Khunti K, Mingrone G, Hopkins D, Binkerfeld A, et al. Practical recommendations for the management of diabetes in patients with COVID-19. Lancet Diabetes Endocrinol. 2020;8:546-50.

56. Ceribelli A, Motta F, De Santinis M, Andari A, Ridgway W, Gershwin M, et al. Recommendations for coronavirus infection in rheumatic diseases treated with biologic therapy. J Autoimmun. 2020;109:102442.

57. Hermans C, Lambert C, Sogorb A, Wittebole X, Belkhir L, Yombi J. In-hospital management of persons with haemophilia and COVID-19: practical guidance. Haemophilia. 2020;26(5):768-72.

58. Beigel JH, Tomashek KM, Dodd LE, et al. Remdesivir for the Treatment of Covid-19 - Final Report. This article was published on October 8 2020, and updated on October 9, 2020, at NEJM.org. DOI: 10.1056/ NEJMoa2007764

59. Cao Y, Wei J, Zou L, Jiang T, Wang G, Chen L, et al. Ruxolitinib in treatment of severe coronavirus disease 2019 (COVID-19). A multicenter, single-blind, randomized controlled trial. J Allergy Clin Immunol. 2020;146(1):137-46.e3.

60. Dimopoulos G, Mast Q, Markou N, Theodorakopoulou M, Komnos A, Mouktaroudi $M$, et al. Favorable anakinra responses in severe Covid-19 patients with secondary hemophagocytic lymphohistiocytosis. Cell Host Microbe. 2020;28(1):117-23.e1.

61. Barnes G, Burnett A, Allen A, Blumenstein M, Clark N, Cuker A, et al. Thromboembolism and anticoagulant therapy during the COVID19 pandemic: interim clinical guidance from the anticoagulation fórum. J Thromb Thrombolysis 2020;50:72-81. DOI: 10.1007/s11239-020-02138-z. 